\title{
How to Restore Rotation Center in Total Hip Arthroplasty for Developmental Dysplasia of the Hip with the Assistance of Three Dimensional Printing Technology? A Pilot Study
}

Heng Zhang ( $\square$ bygkzhangheng@163.com )

The first affiliated hospital of Bengbu Medical College

Yang Liu

The first afffiliated hospital of Bengbu Medical College

Kuanxin Li

The first affiliated hospital of Bengbu medical college

Zheng Zhang

The First Affiliated Hospital of Bengbu Medical Co

Jianzhong Guan

The First Affiliated Hospital of Bengbu Medical Co

Qirong Dong

Second Affiliated Hospital of Soochow University

Jiansheng Zhou

The First Affiliated Hospital of Bengbu Medical Co

Research article

Keywords: rotation center, hip arthroplasty, DDH, 3-D print

Posted Date: May 18th, 2020

DOI: https://doi.org/10.21203/rs.3.rs-26630/v1

License: (c) (i) This work is licensed under a Creative Commons Attribution 4.0 International License. Read Full License 


\section{Abstract}

Background: It is technically challenging to restore hip rotation center exactly in total hip arthroplasty (THA) for patients with end-stage osteoarthritis secondary to developmental dysplasia of the hip (DDH) due to the complicated acetabular morphology changes. In this study, we developed a new method to restore hip rotation center exactly and rapidly in THA with the assistance of three dimensional (3-D) printing technology.

Methods: Seventeen patients (21 hips) with end-stage osteoarthritis secondary to DDH who underwent THA were included in this study. Simulated operations were performed on 3-D printed hip models for preoperative planning. The Harris fossa and acetabular notches were recognized and restored to locate acetabular center. The agreement in the size of acetabular cup and bone defect between simulated operations and actual operations were analyzed.Clinical and radiographic outcomes were recorded and evaluated.

Results: The sizes of the acetabular cup of simulated operations on 3-D printing models showed a high rate of coincidence with the actual sizes in the operations $($ ICC value $=0.930)$ There was no significant difference statistically between the sizes of bone defect in simulated operations and the actual sizes of bone defect in $\mathrm{THA}(\mathrm{t}$ value $=0.03 \otimes \mathrm{P}$ value $=0.97$ ). The average Harris score of the patients was improved from $(38.33 \pm 6.07)$ preoperatively to the last follow-up $(88.61 \pm 3.44)$ postoperatively. The mean vertical and horizontal distances of hip rotation center on the pelvic radiographs were restored to $(15.12 \pm 1.25 \llbracket \mathrm{mm}$ and (32.49 \pm 2.83$) \mathrm{mm}$ respectively. No case presented dislocation or radiological signs of loosening until last follow-up.

Conclusions: The application of 3-D printing technology faciliate orthopedists to recognize the morphology of Harris fossa and acetabular notches, locate the acetabular center and restore the hip rotation center rapidly and acurately.

\section{Background}

It is advocated to restore hip rotation center anatomically in THA for patients with end-stage osteoarthritis secondary to $\mathrm{DDH}$, owing to anatomical reconstoration can recover the balance between prosthesis and soft tissue ,thus reducing the wear rate and prosthesis loosening[1-3].

Yet, there is lack of a intuitive and operable method for the surgeons to locate hip rotation center in THA. With the rapid development of 3-D printing technology, the individual life-size models allow surgeons to simulate operations and perform precise treatment,as well as improve outcomes[4-6]. In this study,we developed a new method to restore the hip rotation center by simulating operations on 3-D printing model.

\section{Methods}

This study was approved by the Ethics Committee on Human Research of the first affiliated hospital of Bengbu Medical College, and informed consents were obtained from all involved patients. The medical 
charts were assessed in terms of the inclusion criteria as below:hip end-stage osteoarthritis (OA Tonnis \stage) due to DDH (Crowe classification type I to IV ), pain affected daily life and work, the muscle strength of the affected limb was normal, age range was from 30 to 75 years old; no hip trauma history and other hip diseases history. The exclusion criteria were hip osteoarthritis(OA Tonnis stage I to $\Downarrow$ ) ,the hip joint pain was not obvious, the muscle strength of the affected limb was below IV class, ages older than 75 years old or younger than 30 years old; hip trauma history or combined with other hip diseases.The study involved 17 patients $₫ 21$ hips $\varangle$ who underwent primary cementless THA from March 2014 to July 2018 . The 17 patients included 4 men $₫ 5$ hips $₫$ and 13 women ( 16 hips). The average age is $58.00 \pm 8.12$ years (range from 45 to 71 years). The average of body mass index is $25.70 \pm 3.33 \mathrm{~kg} / \mathrm{m}^{2}$ (range from 18.55 to $31.63 \mathrm{~kg} / \mathrm{m}^{2}$ ). The average preoperative LLD was $2.43 \pm 1.07 \mathrm{~cm}$ (range from 0.5 to $4.0 \mathrm{~cm}$ ) )in which 7 patients' LLD is below

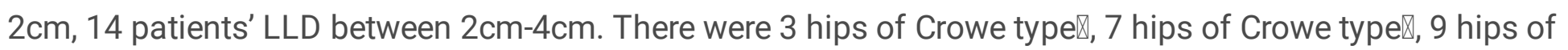

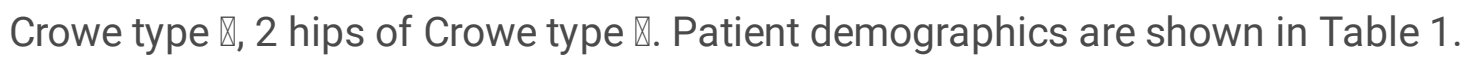

The preoperative pelvic radiographs usually displayed that the acetabulum was superficial flat the femoral head displaced toward outside and upward, the shenton line was interrupted, the joint space was narrow and the surface of Harris fossa was covered with osteophytes(Fig .1A). The false socket and osteophytes could be displayed clearly in 3-D computed tomography (CT) image(Fig. 1B). Although the Harris fossa was covered with osteophytes, but it always existed, which could be judged and discovered by cross sectional CT image(Fig. 1B).The Harris score was used to evaluate the state of preoperative hip joint function.

\section{Simulated operation and Preoperative Planning for restoring hip roation center on 3-D printing model}

Individual life-size model was printed by 3-D printing machine ( Arigin 3-DM400, Shanghai Arigin medical technology Co. LTD 『China) using polylactic acid (PLA) material『based on patients' pelvis 3 D CT data (Fig.2A). The Harris fossa was covered with osteophytes and restored its morphology by removing osteophytes(Fig.2B).The acetabular anterior and posterior notches were recognized and the perpendicular bisector of acetabular anterior and posterior notch line was marked. The acetabular center was loacted at $25 \sim 31 \mathrm{~mm}$ (mean $28.7 \mathrm{~mm}$,depended on the size of the acetabulum) above the intersection point of the perpendicular bisector and acetabular anterior and posterior notch line(Fig.2C), which could also be located by ourself-developed acetabular center locator(Fig.2D) [7]. During the process of acetabular reaming, the anteversion was controlled in the range of $15^{\circ} \pm 10^{\circ}$, while the inclination was $40^{\circ} \pm 10^{\circ}$, and the depth was to bottom of Harris fossa. Reaming was at concentric circles from small to large, aiming at the acetabular center (Fig.2E) $₫$ The final size of the grind acetabulum was determined by the criterion that the anterior and posterior wall had sufficient clamping force to obtain good initial stability of the cup(Fig.2F). The bone defect above the cup was filled with bone wax(Fig.2G). The size of cup and bone defect was recorded. The model of integral customized acetabular prosthesis was designed using UG software (Unigraphics NX by Siemens PLM Software) and printed by 3-D printing machine. The model of integral customized acetabular prosthesis fitted the grind acetabulum exactly (Fig.2H).

The size of bone defect was measured by bone wax method. We only measured the area of contact surface between bone wax and cup, which was vital to determine bone ingrowth(Fig.3A.3B). The surface of bone wax which required to be measured was covered with gauze precisely(Fig.3C). Removed the gauze from the 
bone wax and layed it to be flat. Placed the gauze in the area surrounded by two rulers which were mutually perpendicular(Fig.3-D).Took the picture by digital camera (SONY DSC- T90,10.2 megapixels, SONY Company, Japan). The pictures were imput into Photoshop software. The boundary of gauze and rulers were marked respectively.The size of selected zone could be expressed by the pixels. The actual area of gauze could be calculated by the method: the pixels of gauze zone divided the pixels of rulers zone and then multiplied the actual area of rulers zone,such as $\left(434^{\star} 147\right) \div(737 * 733) \star(10 \star 10) \mathrm{cm}^{2}$.

\section{Surgical Technique}

Posterolateral approach was applied for all patients with lateral decubitus position. The contracture soft tissue around the hip joint was released completely, paying much attention to protect the sciatic nerve and gluteus medius femoral insertion. The elongated and thickened joint capsule was resected and the osteophytes around the acetabulum were removed completely. The true acetabulum could be displayed clearly thereafter (Fig.4A). The morphology of Harris fossa was restored by removing the covered osteophytes (Fig.4B). The acetabular anterior and posterior notches were recognized and the perpendicular bisector of acetabular anterior and posterior notch line was marked. The acetabular center was loacted at $25 \sim 31 \mathrm{~mm}$ (mean $28.7 \mathrm{~mm}$,depended on the size of the acetabulum) above the intersection point of the perpendicular bisector and acetabular anterior and posterior notch line[7](Fig.4C). During the process of acetabular reaming, the anteversion was controlled in the range of $15^{\circ} \pm 10^{\circ}$, while the inclination was $40^{\circ}$ $\pm 10^{\circ}$, and the depth was to the cortical bone of Harris fossa's bottom. Reaming was at concentric circles from small to large ,aiming at the acetabular center(Fig.4D). The final size of the grind acetabulum was determined by the criterion that the anterior and posterior wall had sufficient bone and clamping force to obtain good initial stability of the test cup (Fig.4E). The models of integral customized acetabular prosthesis were implanted into the grind socket to verify the accuracy of simulated operations(Fig.4F).The reconstruction methods of bone defect were determined according to the results of simulated operations. The size of bone defect was measured again by bone wax method.Autograft cancellous bone granules from the trimmed femoral head were used to provide adequate superolateral coverage of the cup only when acetabular bone defect $>10 \%$ (Fig.4G). The cementless press-fit acetabular prothesis was implanted to obtain good initial stability, although additional screws were applied if required.

\section{Postperative Management}

Antibiotics were given for infection prophylaxis within 24 hours. Anticoagulants were used to prevent deep vein thrombosis for 5 weeks. Ankle and quadriceps contraction exercises started from the first day after operation .Patients were made touch-down weight bearing under the asistance of crutches until $4 \sim 6$ weeks ,partial weight bearing until 12 weeks, then to fully weight bearing thereafter.

Patients were followed up clinically and radiologically at 0 week, 6 months and yearly thereafter. Harris score were used to evaluate the recovery of hip joint function. Radiological assessment was performed using the pelvis plain radiograph(Fig.5). The vertical distance from the center of hip rotation to the interteardrop line and the horizontal distance from the center of hip rotation to the ipsilateral teardrop were 
measured, which were used to assess the efficacy of restoring hip rotation center and acetabular cup migration.

\section{Statistical Analysis}

All statistical analyses were performed by using SPSS software for Windows (version 19.0; SPSS, Chicago, IL).Continuous variables were presented as means and ranges and categorical variables as frequencies. The 2-sided paired T test was used for comparison between preoperative and postoperative hip rotation center data measurement on pelvis radio graphs $₫$ bone defect area and Harris hip scores. ICCs were used to analyze the agreement between the size of the cup chosen on the basis of preoperative planning and the actual size in the operation. A P value $<.05$ was considered statistically significant.

\section{Results}

The size of bone defect and reaming acetabulum囚as well as the location of acetabular center during the THA had a high consistency with which in simulated operations on 3-D printing model. The mean sizes of bone defect in simulated operations and THA were $4.58 \pm 2.47 \mathrm{~cm}^{2}$ and $4.55 \pm 2.57 \mathrm{~cm}^{2}$ respectively. There was no significant difference statistically between the sizes of bone defect in simulated operations and the actual sizes of bone defect in THA( $t$ value $=0.03 \otimes P$ value $=0.97$ ). The sizes of the acetabular cup of simulated operations on 3-D printing models showed a high rate of coincidence with the actual sizes in the operations (ICC value=0.930). The measurement results of bone defect area and cup size were shown in Table 2.

All 17 patients were available for clinical and radiological follow-up and an average follow-up time was $18.35 \pm 6.86$ months $₫$ range, $12-36$ months.No revisions were needed. No patient had a infection, hip dislocation, symptomatic deep-vein thrombosis and other complications.

Two patients had a slight limp.The average Harris score of the patients was improved from the preoperation (38.33 \pm 6.07$)$ to the last follow-up (88.61 \pm 3.44$)$, including excellent 12 hips, good 8 hips and fair 1 hip , the difference was statistically significant ( $t$ value $=33.03, P<.05)$.The mean preoperative vertical distance of hip rotation center on the pelvic radiographs was $(40.48 \pm 8.42) \mathrm{mm}$, the mean postoperative one was $(15.12 \pm 1.25 \rrbracket \mathrm{mm}$; the mean preoperative horizontal distance of hip rotation center on the pelvic radiographs was $(41.49 \pm 5.17) \mathrm{mm}$, and the mean postoperative one was $(32.49 \pm 2.83) \mathrm{mm}$. The difference between the preoperative and postoperative vertical and horizontal distances of hip rotation center was statistically significant ( $\mathrm{t}$ value $=13.65,6.99 \otimes \mathrm{P}<.05)$.

\section{Discussion}

It is still a challenge for orthopedists to restore hip rotation center anatomically in primary THA for patients with DDH, due to complicated acetabular changes[8-9]. It has been widely accepted and advocated that acetabular prosthesis should be installed anatomically and the hip rotation center should be restored [1013] . However,there are still lack of a simple, intuitive and operable method to locate hip rotation center during the operation up to now.Therefore, we developed a new method to restore rotation center exactly and 
easily by recognizing and restoring Harris fossa and acetabular notches, which could be used to locate acetabular center,with the assisstance of simulating operations on 3-D printed models.Our results demonstrated there was a high rate of coincidence in the sizes of the acetabular component and bone defect between preoperative planning on 3-D printed model and THA. Compared with preoperative positions , hip rotation center were restored anatomically after the surgery.

It is difficult to locate the rotation center during the operation. Many methods [14-16],such as, template method, concentric circle method of the healthy side, Ranawat method, Pierchon method and Pagnano method, etc, have been used to locate the rotation center of the hip joint .However, the above methods were all determined by two-dimensional image of the pelvis.Andriacchi and Strickland [17] estimated that the hip center would lie $1.5 \pm 2 \mathrm{~cm}$ directly distal to the midpoint of a line connecting the antero-superior iliac spine and the pubic symphysis in the frontal plane. Kirkwood[18] aslo agreed with the above point of view. Although these methods take the hip rotation center as three-dimentional concept into consideration, however, they rely on accurate palpation of bony landmarks and estimation of the distance between them to locate the hip joint center,which have limited value to instruct surgeons to locate the hip rotation center in the operation.Dardenne[19] reported that the hip center could be determined by the Pivot method accurately in computer-assisted (or navigated) orthopedic surgery. Computer-assisted technology could promote the accuracy of locating hip rotation center, but it needs high requirements of devices and technology, which is difficult to be popularized.In previous study, we have reported there was a constant relationship between the acetabular center and Harris fossa, as well as acetabular notches, which could be used to locate the acetabular center. In this study, we found Harris fossa always existed ,even in cases of Crowe type II and $\otimes$, which could be judged and discovered by preoperative acetabulum CT scan.The acetabulum often presented to be dish shape and shell shape in Crowe type II and $\otimes$, while the Harris fossa presented to be closed shape and triangle shape correspondingly. The acetabulum were filled with a large number of ostephytes, which also covered the Harris fossa. During the operation, the Harris fossa could be restored by the following procedures:区exposing and recognizing the acetabulum and Harris fossa by removing the ostephytes. The Harris fossa was suited in the inferior part of the acetabulum, as well in the middle of anterior and posterior wall of the acetabulum. FFinding out the latent gap of Harris fossa by the following two methods. One method was using a small osteotome to hit the middle part of Harris fossa, then an empty feeling could be obtained. The other one was using a small flat nerve dissector to insert into the gap from the lowest part of the Harris fossa.खRestoring the Harris fossa's morphology by using kerrison rongeur to remove the surface cortex bone to expose the whole outline.Once the morphology of Harris fossa was restored, there were two methods helped to locate acetabular center.The first way was to utilize the relationship between the acetabular center and Harris fossa ,as well as acetabular notches. The acetabular center was loacted at $25 \sim 31 \mathrm{~mm}$ (mean $28.7 \mathrm{~mm}$,depended on the size of the acetabulum) above the intersection point of acetabular anterior and posterior notch line and its perpendicular bisector.The second method was to use our self-developed acetabular center locater.We used this method to locate acetabular center in all DDH patients, whose hip rotation center could be restored exactly according to the assessment of postoperative X-rays. 
The reconstruction of acetabular bone defect in THA was important because it affects the initial stability of the acetabular prothesis[11].The size of acetabular bone defect determined the management ways of reconstrution. Many schoolars[11][20-21] reported the acetabular bone defect could be measued by imageological technology. However, there is still lack of an effective method to measure the bone defect in the operation. Here we present a solution for the measurement of acetabular bone defect accurately in THA using bone wax. The bone wax is easy to reshape and adapt to irregular bone defect. The curved suface of bone defect could be transformed to flat area by using the gauze to cover the surface of bone wax. The boundary of irregular gauzes could be outlined easily in Photoshop software. This method realized precise measurement of bone defect area.

Previous studies have reported 3-D printing simulated operations improved the accuracy and safety of orthopedic surgeries [22]. Huang et al[23] demonstrated that 3-D printing technology increased the predictability, accuracy and reliability of both-column acetabular fractures reduction. Wang reported that, compared with CT images or humerus-subtracted volume renderings, the 3-D-printed model was able to represent the glenoid bony morphology accurately and clearly which faciliated shoulder surgeons to perform preoperative planning[24]. A double-blinded randomized clinical trial indicated that osteotomy simulation on the 3-D-printed pelvis model for DDH patients increased the success rate of the operation and shortened surgery time, as well as promoted postoperative recovery[25].Xu et al [26] recommended the use of the 3-D printed model before THA in DDH patients due to better preoperative planning and intraoperative orientation,as well as surgical outcomes.Our study demonstrated the high agreement between the size of the acetabular cup and bone defect of simulated operations on 3-D printing models and the actual size used in the operations.

Our study had several limitations. Firstly, It was an independent study, there was no control group and the sample size was small. Secondly, The elastic attribute of 3-D printed model is not like bone, resulting in the errors between the simulated and intraoperative option of acetabular cup size chosen .Finally, we only measured the contact interface area of bone defect,however, the bone defect was a 3-dimensional concept.

\section{Conclusions}

The Harris fossa and acetabular notches are the important anotomical markers which help to locate acetabular center in THA.The application of 3-D printing technology faciliate orthopedists to recognize the morphology of Harris fossa and acetabular notches, locate the acetabular center and restore the hip rotation center rapidly and acurately.

\section{Abbreviations}

DDH: Developmental dysplasia of the hip;

THA: Total hip arthroplasty;

3-D : Three dimensional; 
LLD: Lower limbs length discrepancy;

CT : Computed tomography;

PLA: Polylactic acid.

\section{Declarations}

\section{Ethics approval and consent to participate}

This study was approved by the Ethics Committee on Human Research of the First Affiliated Hospital of Bengbu Medical College, and informed consent was obtained from all involved patients.

\section{Consent to publish}

This study were well understood and agreed by all the enrolled patients, which was recorded in the consent for publication.

\section{Availability of data and materials}

All data generated or analysed during this study are included in this published article.

\section{Competing interests}

The authors declare that they have no competing interests.

\section{Funding}

This study was supported by the University Natural Science Research Project of Anhui Province(Grant No.KJ2019A0333), the Natural Science Foundation of Bengbu Medical College (Grant No. BYKY18126) and the Science and Technology Innovation Guidance Project of Bengbu city(Grant No. 20180307).

\section{Authors' contributions}

This study was conceived and designed by $\mathrm{HZ}$ and JZ. Data was acquired by $\mathrm{HZ}, \mathrm{JG}, \mathrm{ZZ}$, and JZ. $\mathrm{HZ}$ performed the statistical analysis and drafted the manuscript. The manuscript was edited by $\mathrm{HZ}, \mathrm{YL}, \mathrm{KL}$, and QD. The manuscript was reviewed and approved for final publication by all authors.

\section{Acknowledgements}

None.

\section{References}

[1] Vasileios I. SakellariouखMichael ChristodoulouヌGregory Sasalos, et al. Reconstruction of the acetabulum in developmental dysplasia of the hip in total hip replacement[J]. Arch Bone Jt Surg. 2014;2(3):130-136. 
[2] Zhang Y, Zhu ZA, Zhu MS, et al. Anatomic hip center reconstruction during total hip arthroplasty for severe osteoarthritis secondary to hip dysplasia[J]. Chin J Joint Surg(Electronic Edition),2016;10『4『:393398.

[3] Rasi AM, Kazemian G, Khak M, et al. Shortening subtrochanteric osteotomy and cup placement at true acetabulum in total hiparthroplasty of Crowe III-IV developmental dysplasia: results of midterm followup.Eur J Orthop Surg Traumatol. 2018 ;28(5):923-930.

[4] Huang JH, Liao H, Tan XY, et al.Surgical treatment for both-column acetabular fractures using preoperative virtual simulationand three-dimensional printing techniques.Chin Med J (Engl) . 2020 ;133(4):395-401.

[5] Liu K, Li Z, Ma Y, et al. 3-D-printed pelvis model is an efficient method of osteotomy simulation for the treatment of developmental dysplasia of the hip.Exp Ther Med. 2020;19(2):1150-

[6] Wang KC, Jones A, Kambhampati S, et al. CT-Based 3-D printing of the glenoid prior to shoulder arthroplasty: bony morphology and model evaluation.J Digit Imaging. 2019 ;32(5): 816-826.

[7] Zhang H, Zhou J, Guan J,et al. How to restore rotation center in total hip arthroplasty for developmental dysplasia of the hip by recognizing the pathomorphology of acetabulum and Harris fossa?J Orthop Surg Res. $2019 ; 14(1): 339$.

[8] Sakellariou VI, Christodoulou M, Sasalos G,et al. Reconstruction of the acetabulum in develop mental dysplasia of the hip in total hip replacement.Arch Bone Jt Surg.2014;2(3):130-136.

[9] Baki ME, Timurkaynak A, Aydın H,et al. Metal-on-metal dysplasia cup total hip arthroplasty for hip osteoarthritis secondary to developmental dysplasia of the hip.Eklem Hastalik Cerrahisi. 2014;25(3):154157.

[10]Greber EM, Pelt CE, Gililland JM ,et al.Challenges in total hip arthroplasty in the setting of developmental dysplasia of the hip.JArthroplasty.2017;32(9S):S38-S44

[11]Zhang L, Lu X.Acetabular Cup Positioning during Total Hip Replacement in osteoarthritis secondary to developmental dysplasia of the hip - a Review of the Literature.Acta Chir Orthop Traumatol Cech. 2019;86(2):93-100.

[13] Fukui K, Kaneuji A, Sugimori T, et al. How far above the true anaomic position can the acetabular cup be placed in total hip arthroplasty?Hiplnt.2013;23(2):129-134.

[14] Pierchon F, Migaud H, Duquennoy A, et al. Radiologic evaluation of the rotation center of the hip.Rev Chir Orthop Reparatrice Appar Mot. 1993;79(4):281-284.

[15]Ranawat CS, Dorr LD, Inglis AE. Total hip arthroplasty in protrusio acetabuli of rheumatoid arthritis. J Bone Jt Surg, 1980;62A : 1059-1065. 
[16] Fessy MH, N'Diaye A, Carret JP, et al. Locating the center of rotation of the hip.Surg Radiol Anat. 1999;21(4):247-250.

[17] Andriacchi TP, Strickland AB. Gait analysis as a tool to assess joint kinetics. In: Proceedings of NATO. Advanced Study Institute Biomechanics of Normal and Pathological Articulating Joints. Lisbon, Portugal: NATO, 1983:83-103.

[18]Kirkwood RN, Culham EG, Costigan P.Radiographic and non-invasive determination of the hip joint center location: effect on hip jointmoments.Clin Biomech (Bristol,Avon). 1999 ;14(4):227-235.

[19]Dardenne G, Dib Z, Poirier N, et al.What is

the best hip center location method to compute HKA angle in computer-assistedorthopedic surgery? In silico and in vitro comparison of four methods. Orthop Traumatol Surg Res. 2019;105(1):55-61.

[20] Putzer D, Scholl F, Dankl L, Med Eng Phys,et al.Intraoperative bone defect measurement in the acetabulum using a navigation system: The influence of bone porosity on the digitalization process. $2018 ; 59: 75-80$.

[21]Schierjott RA, Hettich G, Ringkamp A, et al.

A method to assess primary stability of acetabular components in association with bone defects.J Orthop Res. $2020 ; 1: 1-10$

[22]Trauner KB.The emerging role of 3-D printing in arthroplasty and orthopedics.J Arthroplasty.2018;33(8):2352-2354.

[23]Huang JH, Liao $\mathrm{H}$, Tan $\mathrm{XY}$,et al. Surgical treatment for both-column acetabular fractures using preoperative virtual simulationand three-dimensional printing techniques.Chin Med J (Engl). 2020;133(4):395401..

[24]Wang KC, Jones A, Kambhampati S,et al.CT-based 3-D printing of the glenoid prior to shoulder arthroplasty: bony morphology and model evaluation.J Digit Imaging. 2019 ;32(5):816-826.

[25] Liu K, Li Z, Ma Y ,et al.3-D-printed pelvis model is an efficient method of osteotomy simulation for the treatment of developmental dysplasia of the hip.Exp Ther Med. $2020 ; 19(2): 1155-1160$.

[26]Xu J, Li D, Ma RF ,et al. Application of rapid prototyping pelvic model for Patients withDDH to Facilitate ArthroplastyPlanning:A Pilot Study.J Arthroplasty. $2015 ; 30(11)$ :19631970.

\section{Tables}

Table 1 Demographics of Patients. 


\begin{tabular}{|c|c|}
\hline Gender & \\
\hline Male & 4 \\
\hline Female & 13 \\
\hline Age $(y)$ & $58.00 \pm 8.12$ هrange,45-71ه \\
\hline Body mass index $(\mathrm{kg} / \mathrm{m} 2)$ & $25.70 \pm 3.33 \rrbracket$ range,18.55-31.63凶 \\
\hline LLD $₫ \mathrm{~cm} \rrbracket$ & $2.43 \pm 1.07 \rrbracket$ range,0.5-4.0区 \\
\hline Crowe classification & (four patients ,bilateral DDH) \\
\hline Crowe type $\rrbracket$ & 3 \\
\hline Crowe type $\rrbracket$ & 7 \\
\hline 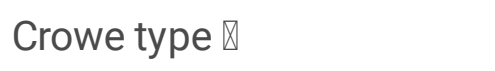 & 9 \\
\hline Crowe type $\otimes$ & 2 \\
\hline
\end{tabular}

Table 2 The measurement results of bone defect area and cup size. 
Patient Gender Age Crowe Preoperative Preoperative Intraoperative Intraoperative No. $\quad$ type (Male/ (year) plancup plan bone cup size bone defect Female】 size defect area $\left(\mathrm{cm}^{2}\right)$ area $\left(\mathrm{cm}^{2}\right)$

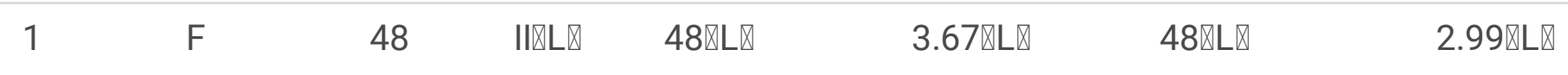

\begin{tabular}{|c|c|c|c|c|c|c|c|}
\hline 2 & $\mathrm{M}$ & 65 & $\mathrm{II} \otimes \mathrm{R} \square$ & $48 \bowtie \mathrm{R} \rrbracket$ & $4.40 \rrbracket \mathrm{R} \rrbracket$ & $50 \rrbracket \mathrm{R} \rrbracket$ & $4.62 \rrbracket \mathrm{R} \rrbracket$ \\
\hline 3 & $\mathrm{~F}$ & 64 & IIVLQ & 48『L》 & 3.97凶L』 & 48『L】 & 3.77凶L囚 \\
\hline 4 & $\mathrm{M}$ & 71 & IIIXLQ & 54囚L】 & $10.96 \rrbracket \mathrm{L} \rrbracket$ & 58هL】 & 11.81هL】 \\
\hline \multirow[t]{2}{*}{5} & \multirow[t]{2}{*}{$\mathrm{F}$} & \multirow[t]{2}{*}{53} & \multirow{2}{*}{$\begin{array}{l}\text { III囚L邓II } \\
\triangle R \rrbracket\end{array}$} & 46『L】 & 4.94】L】 & 48هL】 & 5.12囚L囚 \\
\hline & & & & 46هR囚 & $3.62 \bigotimes \mathrm{R} \rrbracket$ & 46هR囚 & $3.51 \rrbracket \mathrm{R} \rrbracket$ \\
\hline 6 & $\mathrm{~F}$ & 53 & II $\nabla L \bigotimes$ & 48囚L】 & 3.69هL》 & 48『L》 & 3.51凶L》 \\
\hline 7 & $\mathrm{M}$ & 49 & I $\triangle \mathrm{R} \rrbracket$ & $50 \rrbracket R \rrbracket$ & 3.16هR囚 & 50هR囚 & 3.25囚R囚 \\
\hline 8 & $\mathrm{~F}$ & 50 & IIIØL】 & 46囚L】 & 5.35囚L』 & 48هL】 & 5.56هLه \\
\hline 9 & $\mathrm{~F}$ & 61 & IXR】 & $52 \rrbracket \mathrm{R} \rrbracket$ & $3.17 \rrbracket \mathrm{R} \rrbracket$ & 52هR》 & 3.25邓R》 \\
\hline \multirow[t]{2}{*}{10} & \multirow[t]{2}{*}{$\mathrm{F}$} & \multirow[t]{2}{*}{68} & \multirow{2}{*}{ 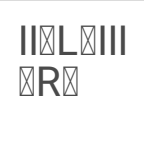 } & $50 \bowtie \mathrm{R} \rrbracket$ & 4.47囚L囚 & 50هL】 & 4.42囚L囚 \\
\hline & & & & $50 \rrbracket L \rrbracket$ & $6.87 \otimes R \rrbracket$ & $50 \rrbracket \mathrm{R} \rrbracket$ & $6.90 \rrbracket \mathrm{R} \rrbracket$ \\
\hline 11 & $\mathrm{~F}$ & 53 & $\mathrm{I} \otimes \mathrm{R} \mathbb{\mathrm { N }}$ & $48 \bowtie \mathrm{R} \rrbracket$ & 2.11هR》 & $48 \bowtie \mathrm{R} \rrbracket$ & 1.98هR \\
\hline \multirow[t]{2}{*}{12} & \multirow[t]{2}{*}{$\mathrm{F}$} & \multirow[t]{2}{*}{53} & IV $₫ \mathrm{~L} \rrbracket$ & $44 \rrbracket \mathrm{R} \bigotimes$ & OهL囚 & 44囚L】 & O凶L】 \\
\hline & & & $\mathrm{IV} \rrbracket \mathrm{R} \rrbracket$ & 44囚L】 & $0 \otimes R \rrbracket$ & 44囚R囚 & $0 \otimes R \rrbracket$ \\
\hline \multirow[t]{2}{*}{13} & \multirow[t]{2}{*}{$\mathrm{M}$} & \multirow[t]{2}{*}{65} & IIXL】 & $48 \bowtie \mathrm{R} \rrbracket$ & 3.56هL】 & 50هL》 & 3.86هL】 \\
\hline & & & $\mathrm{III} \otimes R \otimes$ & 48『L】 & 6.96冈R』 & 48هR囚 & $5.72 \bigotimes \mathrm{R} \bigotimes$ \\
\hline 14 & $\mathrm{~F}$ & 61 & $\mathrm{II} \otimes \mathrm{R} \otimes$ & 46冈R》 & $5.36 ه \mathrm{R} \rrbracket$ & 46هR囚 & 5.31هR》 \\
\hline 15 & $\mathrm{~F}$ & 45 & $\mathrm{III} \triangle \mathrm{R} \otimes$ & 46冈R冈 & $6.42 \bigotimes \mathrm{R} \rrbracket$ & 46هR囚 & $6.52 \rrbracket \mathrm{R} \rrbracket$ \\
\hline 16 & $\mathrm{~F}$ & 69 & $\mathrm{III} \otimes \mathrm{R} \otimes$ & $48 \rrbracket \mathrm{R} \rrbracket$ & 6.69هR》 & $48 \bowtie \mathrm{R} \rrbracket$ & 6.56هR》 \\
\hline 17 & $\mathrm{~F}$ & 58 & $\mathrm{III} \triangle \mathrm{R} \otimes$ & $48 \rrbracket \mathrm{R} \rrbracket$ & 6.81هR》 & 50هR】 & $6.98 \bowtie \mathrm{R} \rrbracket$ \\
\hline
\end{tabular}




\section{Figures}

\section{Figure 1}

(A) Preoperative X-ray . (B)Preoperative 3D-CT. (C) Preoperative horizontal CT scan. 3D=three-dimensional.

\section{2}

Figure 2

(A) 3D printing model. (B) Recognizing and restoring Harris fossa.(C) Locating the acetabular center.(D) Locating the acetabular center by ACL.(E) Reaming the acetabulum.(F) Installing the acetabular cup.(G) Filling and measuring the bone defect by bone wax. $(\mathrm{H})$ Installing the integral customized acetabular prothesis model. $3 \mathrm{D}=$ three-dimensional. $\mathrm{ACL}=$ acetabular center locator.

\section{Figure 3}

(A[B) Bone wax. (C) Using the gauze to cover the surface of bone wax needed to be measured.(D) Laying the gauze to be flat and placing it in the measured region.(E) Measuring the area of gauze by Photoshop software.

\section{Figure 4}

(A) Exposing the acetabulum.(B) Recongnizing and restoring Harris fossa.(C) Locating the acetabular center .(D) Reaming the acetabulum.(E) Installing actabular test model.(F) Installing integral customized acetabular cup model.(G)Installing acetabular prothesis and IBG. 3D=three-dimensional. IBG= impaction bone graft. 


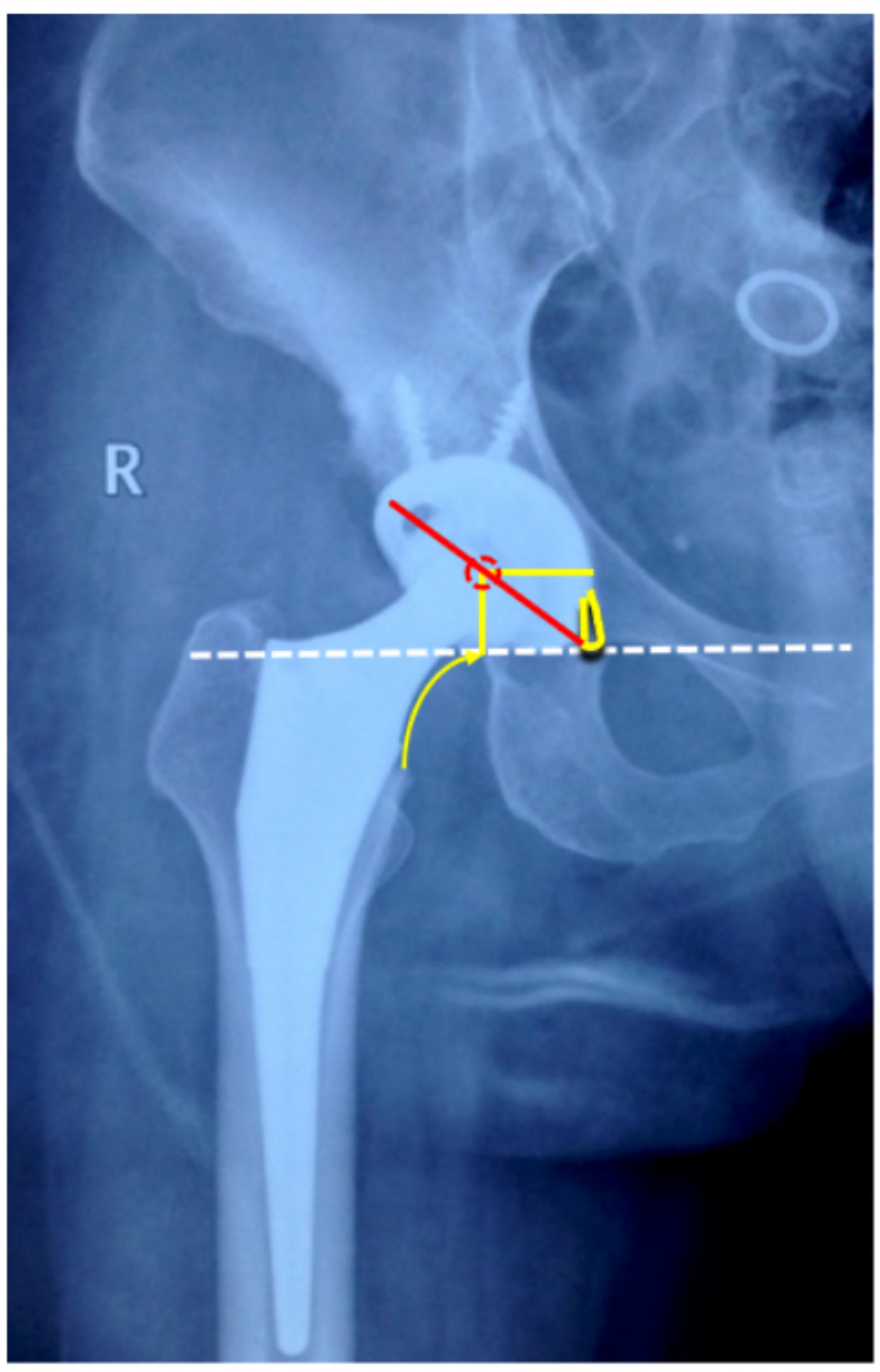

Figure 5

Postoperative X-ray 